Recepción: 25/01/2019

Aceptación: 26/02/2019

Publicación: 05/03/2019

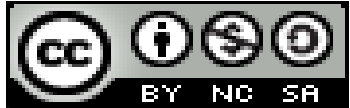

Ciencias económicas y empresariales

Artículo de revisión

\title{
Los factores de los ecosistemas de innovación y sus implicaciones en las incubadoras empresariales
}
The factors of innovation ecosystems and their implications in business incubators

Os fatores dos ecossistemas de inovação e suas implicações nas incubadoras de empresas

\author{
Jazmín Aracelly Arce-Medina ${ }^{\mathrm{I}}$ \\ jarce7239@uta.edu.ec \\ Diana Cristina Morales-Urrutia II \\ dc.moralesu@uta.edu.ec \\ Pilar Lorena Rivera-Badillo ${ }^{\mathrm{III}}$ \\ pl.rivera@uta.edu.ec
}

Correspondencia: jarce7239@uta.edu.ec

${ }^{\text {I }}$ Estudiante en Organización de Empresas de la Universidad Técnica de Ambato. Ambato, Ecuador.

${ }^{\text {II }}$ Doctora dentro del Programa de Desarrollo Económico E Innovación, Máster Universitario en Desarrollo Económico e Innovación en la Especialidad de Gestión de la Investigación y de la Innovación, Ingeniera Comercial con Mención en Administración de la Productividad, Docente de la Facultad de Ciencias Administrativas de la Universidad Técnica De Ambato. Ambato, Ecuador.

III Doctor dentro del Programa de Doctorado de Economía de la Empresa y Finanzas, Máster Universitario en Organización se Empresas, Magíster en Docencia y Currículo para Paz Educación Superior, Licenciada en Contabilidad y Auditoría Contador Público Autorizado, Docente de la Facultad de Ciencias Administrativas de la Universidad Técnica De Ambato. Ambato, Ecuador. 


\title{
Resumen
}

Para el desarrollo de las innovaciones empresariales es fundamental contar con un ecosistema enfocado en fortalecer las relaciones que influyen en la evolución de la actividad emprendedora acorde a las necesidades de una región. El objetivo de esta investigación fue determinar los factores relevantes en un ecosistema de innovación basados en el análisis de ocho países (Alemania, España, Reino Unido, Estados Unidos, México, Brasil, Chile y Ecuador). Se utilizó una metodología cualitativa para el análisis de los diferentes conceptos de ecosistemas de innovación, así como los factores del entorno y su participación en las incubadoras empresariales, y cuantitativa por el estudio histórico de 19 variables consideradas en la encuesta realizada por el Global Entrepreneurship Monitor. (GEM) en el periodo 2000-2013. Empleando la técnica ANOVA se estableció que entre los factores más significativos están la tecnología, cultura y experiencia empresarial.

Palabras clave: Ecosistemas; emprendimiento; incubadora; innovación

\begin{abstract}
For the development of business innovations it is essential to have an ecosystem focused on strengthening the relationships that influence the evolution of entrepreneurial activity according to the needs of a region. The objective of this research was to determine the relevant factors in an innovation ecosystem based on the analysis of eight countries (Germany, Spain, United Kingdom, United States, Mexico, Brazil, Chile and Ecuador). A qualitative methodology was used to analyze the different concepts of innovation ecosystems, as well as environmental factors and their participation in business incubators, and quantitative by the historical study of 19 variables considered in the survey conducted by the Global Entrepreneurship Monitor. (GEM) in the period 2000-2013. Using the ANOVA technique, it was established that technology, culture and business experience are among the most significant factors.
\end{abstract}

Keywords: Ecosystems; entrepreneurship; incubator; innovation 


\section{Resumo}

Para o desenvolvimento de inovações de negócios é essencial ter um ecossistema focado em fortalecer as relações que influenciam a evolução da atividade empreendedora de acordo com as necessidades de uma região. $\mathrm{O}$ objetivo desta pesquisa foi determinar os fatores relevantes em um ecossistema de inovação baseado na análise de oito países (Alemanha, Espanha, Reino Unido, Estados Unidos, México, Brasil, Chile e Equador). Uma metodologia qualitativa quantitativa para a análise dos diferentes conceitos de ecossistemas de inovação e fatores ambientais e seu envolvimento em incubadoras de empresas, e foi utilizado pelo estudo histórico de 19 variáveis consideradas na pesquisa do Global Entrepreneurship Monitor (GEM) no período 2000-2013. Usando a técnica ANOVA, foi estabelecido que a tecnologia, a cultura e a experiência empresarial estão entre os fatores mais significativos.

Palavras-chave: Ecossistemas; empreendedorismo; incubadora; inovação

\section{Introducción}

Las incubadoras son estructuras de soporte para crear empresas innovadoras, a las que proveen de conocimiento, información y servicios. Estos centros están diseñados para acelerar el crecimiento y éxito de los emprendedores a través de conexiones que ayudan a establecer asociaciones estratégicas y oportunidades comerciales. Los nuevos empresarios utilizan las incubadoras para construir relaciones con los proveedores de recursos externos dispuestos a compartir información, tecnología y finanzas en un entorno determinado (Apa, Grandinetti, \& Sedita, 2017; Kumar, Kumar, Haque, Chowdhury, \& Islam, 2017).

La relación entre diferentes actores participantes de las actividades innovadoras da lugar al ecosistema de innovación el mismo que resulta de una combinación de organizaciones, instituciones, empresarios y recursos necesarios para la innovación y el emprendimiento (Malecki \& Spigel, 2017).

Teniendo en cuenta lo previamente mencionado, es necesario conocer el entorno en el que se desenvuelven los emprendedores, así como los factores relevantes dentro de los ecosistemas de innovación y la relación que tienen con las incubadoras empresariales. 
El propósito del presente artículo es analizar los factores de los ecosistemas de innovación, además de la relación de éstos con las incubadoras empresariales, considerando el efecto en países desarrollados y en vías de desarrollo. El estudio es descriptivo por lo que se realizó una investigación bibliográfica y documental sobre los factores del ecosistema. Desde el punto de vista empírico se utiliza información oficial del Global Entrepreneurship Monitor (GEM) para ocho países Alemania, España, Reino Unido, Estados Unidos, México, Brasil, Chile y Ecuador, con el fin último de determinar los factores con mayor influencia en los ecosistemas de innovación de dichos países.

El estudio se estructura de la siguiente manera. La primera parte, describe la metodología utilizada y los datos recolectados de la base de datos GEM con su interpretación respectiva En la segunda parte se realiza una revisión literaria de los ecosistemas de innovación, sus elementos y factores, seguidamente se menciona la situación de los países desarrollados y en vías de desarrollo, y finalmente se detallan los resultados alcanzados sobre los ecosistemas de innovación en Alemania, España, Reino Unido, Estados Unidos, México, Brasil, Chile y Ecuador.

\section{Materiales y Metodología}

Esta investigación utiliza una metodología cualitativa ya que se analizaron los diferentes conceptos de ecosistemas de innovación, así como los factores del entorno y su participación en las incubadoras empresariales, y cuantitativa, por el estudio histórico de 19 variables consideradas en la encuesta realizada por el Global Entrepreneurship Monitor.

Los datos secundarios se obtuvieron de los resultados de la Encuesta Nacional de Expertos (NES) realizada por el GEM a la Población Adulta (APS) de los 8 países analizados (Alemania, España, Reino Unido, Estados Unidos, México, Brasil, Chile y Ecuador), el periodo estudiado es del 2000 al 2013. La selección del periodo de análisis corresponde a que el GEM publica los resultados completos, para este caso, tres años más tarde de haberse realizado la encuesta (GEM Coordination Team, 2002).

Para verificar los resultados obtenidos se realiza un análisis de estimación en términos de regresión múltiple, para determinar los factores más relevantes de los ecosistemas de innovación y que influyen en la creación de empresas. 
Asimismo, se ha llevado a cabo la prueba de hipótesis a través de ANOVA que determina si el modelo de Regresión esta correlacionado y es factible, además, se complementa con el estadístico de Durbin Watson que utiliza como referencia la significancia, ésta debe ser mayor al nivel de error, y el valor del coeficiente debe ser $>1$, mientras más cercano se encuentre a 2 , más factible es el modelo.

\section{Desarrollo}

\section{Generalidades de los ecosistemas de innovación}

El término ecosistema de innovación se deriva del concepto de ecosistemas de negocios, James Moore fue quien introdujo este concepto en 1993 a través del artículo Predators and Prey: A New Ecology of Competition publicado en Harvard Business Review (Garzón et al., 2015), el articulo sugiere que la empresa se puede ver como parte de un ecosistema de negocios relacionada con una variedad de industrias. Este concepto fue adoptado con facilidad en medios empresariales y gubernamentales, actualmente se utiliza en el mundo de los negocios para referirse a ámbitos territoriales, económicos, industriales o empresariales (Carrillo \& Contreras, 2015).

De acuerdo con Vincens y Grullón (2011) los ecosistemas de innovación nacen en Estados Unidos y aparecieron en América Latina y el Caribe hace 15 años aproximadamente. El ecosistema más reconocido a nivel mundial es el de Silicon Valley de la universidad de Stanford, otros ecosistemas reconocidos son los de las universidades de Babson y Georgia Tech $(\mathrm{H}$. V. Ochoa, Hinojosa, \& Hinojosa, 2018; Vincens \& Grullon, 2011).

Un ecosistema está integrado por organizaciones, instituciones e individuos que apropiadamente, combinados y fortalecidos, son óptimos para la innovación y el crecimiento económico, además promueven el espíritu emprendedor (Arenal, Armuña, Villaverde, \& González, 2018; Malecki \& Spigel, 2017). También, dichos ecosistemas combinan aspectos primordiales tales como: políticos, económicos, sociales y culturales (Smith, 2018).

Otra percepción de los ecosistemas de innovación es que están formados por un conjunto variado de organizaciones y recursos que contribuyen con la innovación dentro de una economía, los mismos están gobernados y orientados por políticas pertenecientes a cada región (FernándezEsquinas, Oostrom, \& Pinto, 2017; Witte, Slack, Keesman, Jugie, \& Wiegmans, 2017). 
Autores como Freeman (1974), Nelson (1993), Patel y Pavitt (1994), Metcalfe (1995), afirman que el Sistema Nacional de Innovación (SNI) es una red de instituciones públicas y privadas, cuya interacción determina el desempeño innovador de la empresa en un país o región, su estructura de incentivos y sus competencias determinan el nivel y la orientación del aprendizaje tecnológico o el volumen y la composición de las actividades generadoras de cambios de un país. Asimismo, proveen el marco dentro del cual los gobiernos deben diseñar e implementar políticas dirigidas a estimular procesos de innovación (Rincón, 2004).

En la misma línea, Lundvall (1992) manifiesta que el SNI es un conjunto de elementos y relaciones que interactúan en la producción, propagación y aplicación del conocimiento nuevo y útil desde el punto de vista económico (Rincón, 2004). Según Moore (1993) el ecosistema es el entorno en el que las necesidades de los clientes son satisfechas por la interacción competitiva y cooperativa entre compañías de distintos sectores (Arenal et al., 2018). Este ecosistema crea productos y servicios para los consumidores, la función principal es hacer posible el desarrollo tecnológico y la innovación (Carrillo \& Contreras, 2015).

El objetivo de los ecosistemas es desarrollar el espíritu emprendedor, promoviendo que los resultados de una investigación sean llevados a cabo y se conviertan en creación y desarrollo de empresas a través de la transferencia de conocimiento, generando así desarrollo económico y captación de inversión necesaria para el desarrollo de una región sustentable (Flores, 2017). La prioridad de los ecosistemas es eliminar la brecha tecnológica y de desempeño económico existente entre empresas y países (Ríos \& Hernández, 2003).

Para Moore (1999) el tamaño y número de actores del ecosistema define el alcance del mismo, entre los integrantes del entorno se encuentran quienes conforman el sistema de valor en el que se desarrollan las actividades empresariales, como proveedores directos e indirectos, distribuidores y clientes. Son parte también del ecosistema los propietarios, socios e inversores, aliados estratégicos y sindicatos. Los competidores o negocios similares se integran igualmente al ecosistema, tal como, las agencias gubernamentales y los organismos de normalización y regulación. Complementan el entorno, los actores que promueven el desarrollo empresarial como el gobierno, instituciones de educación, financieras y capital humano (Garzón et al., 2015). 
Dentro del ecosistema de innovación es fundamental la forma en que sus actores interactúan para producir, difundir y usar el nuevo conocimiento e información que sea económicamente útil (Ríos \& Hernández, 2003). De acuerdo con Crevoisier (2004) el medio innovador combina tres modelos, (1) tecnológico, centrado en la innovación y el aprendizaje, (2) organizacional, refiere a la importancia de las redes y la cooperación entre empresas, y, (3) territorial, enfatiza la proximidad y competencia regional (Nicolopoulou, Karatas- Ozkan, Nummann, \& Vas, 2015).

En base a lo analizado previamente se determina que la relación existente entre los ecosistemas de innovación y las incubadoras, es esencial para el desarrollo empresarial, puesto que ambos se involucran activamente con los factores del medio innovador, por ejemplo las universidades, elemento del ecosistema de innovación, conjuntamente con las incubadoras impulsan al desarrollo de proyectos innovadores, y transformar ideas en negocios, brindando las herramientas necesarias para reducir la tasa de mortalidad empresarial (Mahlmann, Rodríguez, Ferrari, \& Bueno, 2014).

\section{Factores de los ecosistemas de innovación}

Los factores principales de un entorno favorable al emprendimiento y la innovación de acuerdo con el GEM son: acceso a financiamiento, políticas gubernamentales, nivel de educación, investigación y desarrollo, trasferencia tecnológica, normas sociales y culturales (Camino \& Aguilar, 2017). De la misma manera Edquist y Lundvall (1993) sugieren que pueden ser similares en aspectos como el lenguaje, cultura, niveles y formas de vida, patrones de consumo, tamaño del sector público y fortaleza de los tratados comerciales (Ríos \& Hernández, 2003).

En relación a la literatura revisada, varios autores concuerdan en que los factores que incentivan la innovación y el emprendimiento en un ecosistema de innovación, además de fortalecer las relaciones entre los participantes de una incubadora, son: Educación, Parques científicos, Políticas, Programas Gubernamentales y Tecnología (Tabla 1); estos serán estudiados a continuación. 
Tabla 1. Factores del Ecosistema de Innovación

\begin{tabular}{ll}
\hline Autor & Descripción \\
\hline Camino \& Aguilar, 2017 & $\begin{array}{l}\text { Acceso a financiamiento, políticas gubernamentales, nivel de } \\
\text { educación, investigación y desarrollo, transferencia tecnológica, } \\
\text { normas sociales y culturales. }\end{array}$ \\
Smith, 2018 & Elementos sociales, políticos, económicos y culturales. \\
Ngongoni, Grobbelaar, \& Schutte, & $\begin{array}{l}\text { Mercados accesibles, capital humano, financiamiento, cultura y } \\
\text { política. }\end{array}$ \\
Flores, 2017 & Academia, la industria y el gobierno. \\
H. V. Ochoa et al., 2018 & Participación del estado, academia, empresa y la sociedad \\
Garzón et al., 2015 & $\begin{array}{l}\text { Marco regulatorio; valores, cultura y habilidades; acceso a } \\
\text { financiamiento, el mercado, investigación, desarrollo y tecnología; y, } \\
\text { las condiciones naturales y geográficas. }\end{array}$ \\
\hline
\end{tabular}

Fuente: Elaboración propia basada en (Camino \& Aguilar, 2017; Flores, 2017; Garzón et al., 2015; Ngongoni,

Grobbelaar, \& Schutte, 2017; H. V. Ochoa et al., 2018; Smith, 2018)

\section{Educación}

Leydesdorff (2006) plantea el modelo “Triple Hélice” el mismo que relaciona la academia, empresa y gobierno como elementos de la competitividad de una región. Referente a la academia el autor expresa que el recurso humano se forma para generar y transferir conocimientos, así puede promover el emprendimiento y la innovación (Flores, 2017). La calidad de educación en el capital humano genera emprendedores innovadores (Lederman, Messina, Pienknagura, \& Rigolini, 2014).

Casado, Siluk y Zampieri (2012)consideran que las universidades son agentes adecuados para generar ideas y acciones, que buscan resolver situaciones de varias áreas y segmentos de la sociedad (Ribeiro, Vieira, Bent, Lima, \& Meister, 2015). Éstas permiten a los empresarios fomentar el espíritu empresarial a través de las incubadoras, a través del desarrollo de proyectos innovadores, transformando ideas en negocios y contribuyendo a reducir la tasa de mortalidad de las empresas (Mahlmann et al., 2014).

La propiedad intelectual es una manera de proteger el conocimiento del emprendedor, puesto que el conocimiento tiene características de bienes públicos, como la no rivalidad; es decir, el nuevo conocimiento puede ser usado por todos; $y$, la no exclusión que se refiere a que no es posible impedir que otros usen un bien para generar ingresos, esto ha ocasionado desmotivaciones para los emprendedores, por lo que se establecen los derechos de propiedad intelectual como patentes para que el innovador pueda apropiarse de sus conocimientos y comercializarlos (Gauipatín \& Schwartz, 2014). 


\section{Parques científicos}

Los parques científicos son un soporte empresarial que permite la transferencia tecnológica y estimula la creación, incubación y desarrollo de ideas innovadoras, proporcionan a sus usuarios servicios inmobiliarios junto con servicios de empresas, además son mecanismos que facilitan el éxito de las empresas y el crecimiento económico. Estos parques se apoyan con el financiamiento de universidades, gobiernos locales y asociaciones, desempeñan también un papel importante dentro de un ecosistema innovador, empleando tecnologías y conocimientos, aprovechan los resultados de las investigaciones científicas y facilitan el crecimiento de las empresas creadas específicamente en el área de investigación (Salvador, 2008).

Adicionalmente, promueven el emprendimiento, facilitan las relaciones entre las empresas pequeñas, así como el acceso a financiamientos mediante inversores ángeles, fondos de capital o inversores de riesgo, además ofrecen espacios físicos para trabajar (Wood, Wilson, \& García, 2014)

\section{Políticas}

De acuerdo con Edquist y Lundvall (1993), la mayor parte de las políticas públicas influyen en el SNI y a la economía en su conjunto, mejoran el desarrollo económico y tecnológico del país y al mismo tiempo mejoran el prestigio de las empresas y facilitan la innovación (Martínez, González, \& Nieto, 2015; Ríos \& Hernández, 2003).

El reporte de The Economist Intelligence Unit (2014) recomienda varias políticas para crear un ecosistema de innovación exitoso: incrementar la colaboración transfronteriza, construir centros de innovación y espacios de investigación para asegurar un entorno regulatorio amigable, disminuir las barreras a la innovación mediante incentivos financieros al emprendimiento, promocionar el éxito de empresarios nacionales para fomentar la cultura emprendedora, entre otras (Carrillo \& Contreras, 2015). 


\section{Programas gubernamentales}

El apoyo del gobierno es importante para el emprendimiento y la innovación mediante el fortalecimiento de capital humano, la inversión en laboratorios científicos, apoyos a proyectos de I $+\mathrm{D}+\mathrm{i}^{1}$ que impulsan el desarrollo regional (Ramírez \& García, 2010).

El soporte gubernamental permite que las empresas locales crezcan más rápido, por lo que los incentivos ofrecidos por el gobierno deben orientarse a políticas para investigadores y emprendedores, creando estrategias de innovación en áreas que beneficien al desarrollo empresarial, por ejemplo: el soporte financiero incrementa el número de emprendedores (Block, Fisch, \& Praag, 2017; Ramírez \& García, 2010; Wang, 2018).

\section{Tecnología}

Es un conjunto de conocimientos científicos, empíricos, habilidades, experiencias, necesarios para producir, distribuir, comercializar y utilizar bienes o servicios (Ochoa, Quevedo, \& Valdés, 2007).

Además, permite el intercambio de ideas, la internacionalización y colaboración entre agentes económicos permitiendo a los emprendedores que sus ideas más innovadoras y creativas sean ejecutadas con éxito. Por tanto, para que una innovación sea exitosa debe estar conectada con los demás factores del entorno (De los Ríos, Rodríguez, \& Sáenz-Díez, 2015).

El dominio de la tecnología determina la capacidad competitiva de una empresa en productos, servicios y en operaciones, por lo que el éxito empresarial depende de la manera en la que se utiliza los conocimientos y la forma de adaptarse a las situaciones del entorno para así lograr alianzas estratégicas que permitan complementar procesos (Graham, 2006; Ochoa et al., 2007).

\section{Ecosistemas de innovación en países desarrollados y en vías de desarrollo}

Las economías desarrolladas aumentan la transferencia de conocimiento entre universidades e industrias para fortalecer los beneficios sociales. En el estudio realizado en Reino Unido sobre la transferencia de conocimiento universidad - industria, Rossi (2010) revela que las empresas

\footnotetext{
${ }^{1}$ Investigación, desarrollo e innovación
} 
realizan innovaciones frecuentes para aumentar su valor rápidamente en los mercados en movimiento y son más propensas a trabajar con universidades (Mahlmann et al., 2014).

Por otro lado, en las economías emergentes, el SNI, se vincula con la réplica, que no es más que la adaptación e imitación de tecnologías establecidas por las naciones desarrolladas. En estos países se pueden encontrar comunidades locales, regionales, tipo clúster, pero predominan las empresas medianas y grandes, y aglomeraciones de empresas incorporadas a cadenas de valor globales por medio de vínculos o relaciones de dependencia. Estas redes han dado lugar a actualizaciones notables, las que ayudan a la disminución de la brecha tecnológica respecto a los países desarrollados (Ríos \& Hernández, 2003).

Con respecto a lo antes expuesto, se analizan algunos países con economías desarrolladas y en vías de desarrollo.

\section{Alemania}

En Berlín, las universidades, las organizaciones de investigación, centros tecnológicos e incubadoras de negocios brindan condiciones adecuadas para desarrollar nuevas ideas, los inversores ángeles, incubadoras empresariales y universitarias, aceleradoras e inversionistas de capital riesgo apoyan al emprendimiento (Berlin Partner for Business and Technology, 2018). Una de las características es la descentralización del mercado, los costos moderados y la capacidad de atracción de talento extranjero (ICEX, 2017).

\section{España}

Se considera como elementos del sistema de innovación el entorno económico y productivo de la innovación, actividades de innovación científica, el papel de las empresas innovadoras y la política científica y tecnológica, el sistema se consolida mediante el aumento de recursos, cambios institucionales y la interacción entre empresas (Buesa, 2006).

\section{Reino Unido}

Londres es el ecosistema más desarrollado en Europa, esto se debe a la normativa que apoya al emprendimiento, el Fondo de Innovación Regional (RIF), las infraestructuras de soporte como 
aceleradoras, incubadoras y ferias, la cooperación de centros de investigación y empresas privadas (ICEX, 2017).

\section{Estados Unidos}

Uno de los mejores ecosistemas de innovación se encuentra en Los Ángeles, y cuenta con: startup, incubadoras, aceleradoras, capital de riesgo, inversionistas ángeles, venture capital, coworking spaces, consultoras, centros de investigación y desarrollo, centros de innovación de universidades y hackerspace (O. C. de C. en L. Á. ProChile, 2014).

\section{México}

Los principales actores del ecosistema de innovación y emprendimiento en México son: (1) Asociaciones privadas y empresariales vinculadas a Innovación y Emprendimiento, (2) Incubadoras y aceleradoras de negocios, (3) Firmas de Venture Capital e Inversionistas Ángeles, (4) Centros de investigación vinculados al quehacer empresarial, (5) Otras instituciones y redes de apoyo público y privado a la innovación y emprendimiento (ProChile, 2014).

\section{Brasil}

Para el gobierno de este país, un aspecto importante para generar emprendimientos innovadores es el conocimiento, por lo cual ha creado incentivos como cursos técnicos, capacitaciones a docentes, entre otros. Mediante estos programas de apoyo el gobierno ha logrado mejorar la economía brasileña (Mahlmann et al., 2014).

\section{Chile}

La política a largo plazo sobre el emprendimiento ha generado el incremento de fondos $\mathrm{y}$ emprendedores (Cruz \& Arrieta, 2017), CORFO y Startup Chile son iniciativas que han ayudado a la transformación productiva tratando de retener más talentos y empresas en este país. Su objetivo es cerrar la brecha de financiamiento en etapas tempranas mejorando los mecanismos para la creación de empresas (OECD, 2016).

\section{Ecuador}

Los factores que favorecen al entorno innovador es la educación formal, el acceso a tecnologías, normas culturales y de identidad ecuatoriana, mientras que los elementos que afectan al 
emprendimiento son el apoyo financiero y las políticas gubernamentales (Camino \& Aguilar, 2017)

Una vez analizadas las diferentes realidades del entorno innovador en los países de estudio se determina que el ecosistema de innovación difiere en varios aspectos en cada región, los países desarrollados cuentan con mayor número de programas de apoyo consolidados y enfocados en asistir a los nuevos empresarios, mientras que en los países en desarrollo estos incentivos aún están en progreso con la creación de políticas en favor del emprendimiento y la creación de centros de innovación.

\section{Resultados}

De acuerdo a lo expuesto anteriormente, se realiza un análisis empírico basado en los resultados de la encuesta aplicada por el GEM, en donde se consideraron 19 variables que describen factores políticos, gubernamentales, de educación y tecnológicos de cada país en estudio. Cada variable estudiada por el GEM tiene un valor y para efectos de éste análisis se ha utilizado cuatro escalas: favorable, muy favorable, desfavorable, y muy desfavorable, obteniendo así los factores más relevantes para un ecosistema de innovación. En la Tabla 2 se muestran los resultados.

Tabla 2. Factores del ecosistema de innovación

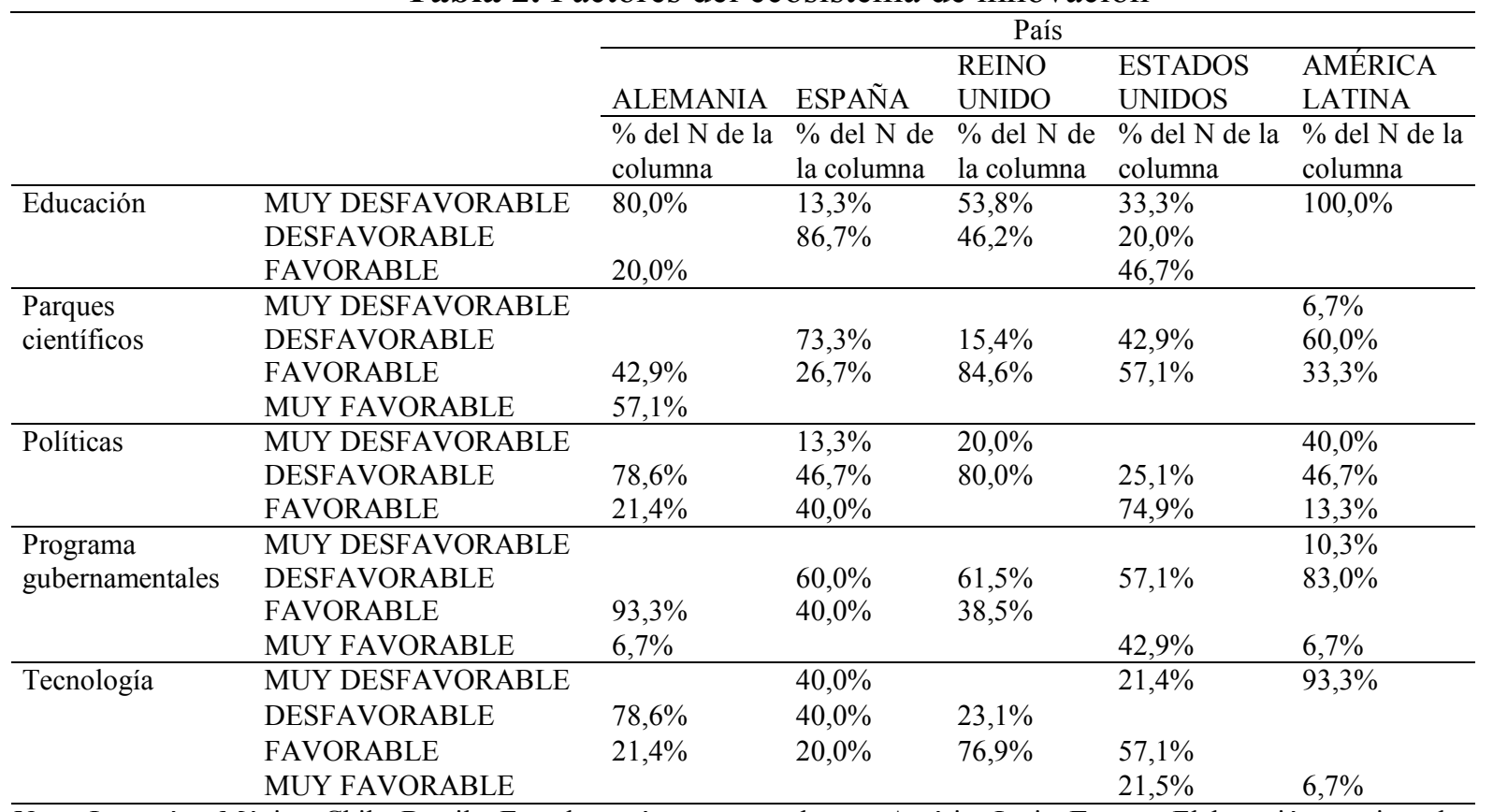

Nota: Los países México, Chile, Brasil y Ecuador están representados por América LatinaFuente: Elaboración propia en base a la encuesta realizada por el GEM 2000-2013 
En Europa, se aprecian algunos factores favorables y muy favorables al ecosistema de innovación, es así que en el caso de Alemania los programas gubernamentales y parques científicos son percibidos como elementos favorables con un $100 \%$, este hecho está vinculado al apoyo del gobierno hacia la investigación por medio de proyectos públicos, y además su sistema de innovación está basado en la cooperación de universidades, empresas e institutos de investigación, que en conjunto crean programas de apoyo al emprendimiento y a la generación de investigación (Pocorey \& Ayabe, 2016). Por su parte, Reino Unido percibe como únicos aspectos favorables la presencia de los parques científicos y la tecnología debido a que éstos brindan apoyo a las empresas de nueva creación y además cuentan con el Fondo de Innovación Regional (RIF) que apoya en la gestación y creación de actividades económicas innovadoras (Salvador, 2008).

Por otro lado, se analizan los factores que no influyen en el ecosistema, entre estos: las variables educación y tecnología no muestran un efecto favorecedor para el entorno alemán, debido a que en este país la educación para el emprendimiento recibe malas calificaciones, de manera similar se muestra la tecnología puesto que de doce aspectos evaluados, ocho se encuentran debajo del valor medio de los países basados en innovación (Sternberg, Wallsich, Gorynia-Pfeffer, Von Bloh, \& Baharian, 2017). De entre las variables de estudio, España se muestra con porcentajes desfavorables que oscilan entre el 70 y el $100 \%$; hecho que puede ser explicado por los altos niveles de burocracia en los procesos administrativos y el exceso de impuestos que enfrentan los emprendedores (Peña, Guerrero, González, \& Montero, 2017).

Un panorama relativamente más favorable frente a los otros países analizados, corresponde a los Estados Unidos, en donde las políticas gubernamentales $(74,9 \%)$ y tecnología $(78,6 \%)$, son los factores de mayor relevancia, en este país en particular se impulsa la estructura científico tecnológica para desarrollar productos/procesos apoyados en espacios aptos para la innovación (Ramírez \& García, 2010; Wood et al., 2014). Mientras que los factores que no aportan en el desarrollo empresarial son los programas gubernamentales $(57,1 \%)$ y educación $(53,3 \%)$.

América Latina en conjunto presenta resultados similares a los mostrados por España; es decir, la mayor parte de los factores examinados son considerados desfavorables para el entorno 
innovador de dicha región. Lo antes mencionado puede deberse a que los programas y las políticas diseñadas, en la mayoría de los casos, no están ajustados a la realidad de los países ya que no contempla las características y capacidades únicas que cada país posee, resultando en simples copias de lo aplicado por las naciones desarrolladas (Kantis, 2004).

En concordancia con los resultados encontrados se determina que cada país tiene diferentes impulsores para el emprendimiento, esto se debe a que los empresarios se desenvuelven en escenarios diferentes de culturas, políticas y educación.

Para determinar cuál de los factores estudiados es el más favorable para el entorno, se presenta el análisis de Regresión (R) (Tabla 3), y se observa que hay una $R$ de $84,2 \%$, éste indica que las evidencias estadísticas de los factores políticos, tecnológicos, de educación, programas gubernamentales y parques científicos influyen en los países al momento de generar emprendimientos dentro de un determinado entorno. Adicionalmente en el análisis de coeficiente de determinación o $\mathrm{R}$ cuadrado se obtiene un valor de 70,9\% el cual está relacionado con la existencia de una variabilidad de estos factores según el país de estudio y un cambio en estos indicadores influye directamente al momento de generar ecosistemas de innovación.

El coeficiente de determinación establece la proporción de la variabilidad que se explica en la regresión múltiple

$$
r^{2}=\frac{S C R}{S C T}=\frac{S C T-S C E}{S C T}=1-\frac{S C E}{S C T}
$$

En donde:

SCT: suma de cuadrados total $\Sigma\left(y_{i}-\bar{y}\right)^{2}$

SCR: suma de cuadrados de la regresión $\Sigma\left(\hat{y}_{i}-\bar{y}\right)^{2}$

SCE: suma de cuadrados del error $\Sigma\left(y_{i}-\hat{y}_{i}\right)^{2}$ 
Tabla 3. Análisis de Regresión - Resumen del modelob

\begin{tabular}{|c|c|c|c|c|c|}
\hline Modelo & $\mathrm{R}$ & R cuadrado & $\begin{array}{l}\text { R cuadrado } \\
\text { corregida }\end{array}$ & $\begin{array}{c}\text { Error típ. de la } \\
\text { estimación }\end{array}$ & $\begin{array}{l}\text { Durbin- } \\
\text { Watson } \\
\end{array}$ \\
\hline 1 & $0,842^{\mathrm{a}}$ & 0,709 & 0,606 & 2,59073 & 1,182 \\
\hline
\end{tabular}

a. Variables predictoras: (Constante), educación, políticas, programas gubernamentales, parques científicos, tecnología

b. Variable dependiente: AÑO

Utilizando la herramienta ANOVA (Tabla 4) se resuelve que todos los factores coadyuvan a generar ecosistemas de innovación, según el contexto donde se apliquen. Por lo tanto, se acepta la hipótesis nula, demostrada con la prueba de significancia $(0,000)$, dato que evidencia la confiabilidad de la investigación.

\section{Hipótesis}

$\mathrm{H}_{0}$ : Todos los factores coadyuvan a generar ecosistemas de innovación

$\mathrm{H}_{1}$ : Al menos uno de los factores coadyuva a generar ecosistemas de innovación

\section{Estadístico de la F}

$$
\begin{aligned}
& F\left\{\begin{array}{c}
H_{0}: \beta_{1}=\beta_{2}=\cdots \beta_{p}=0 \\
H_{1}: \text { Uno o mas de los parametros no es cero }
\end{array}\right. \\
& F_{c}=\frac{C M R}{C M E} \begin{array}{c}
\alpha=0,05 \text { es el nivel de significacion de la prueba } \\
\text { CMT }=\frac{\text { SCE }}{p} y S C E /(n-p-1)
\end{array}
\end{aligned}
$$

Se rechaza $\mathrm{H}_{0}$ si el p-valor de $\mathrm{F}_{\mathrm{c}}$ es menor que $\alpha$.

Tabla 4. Anova

\begin{tabular}{ccccccc}
\hline \multirow{2}{*}{ Modelo } & $\begin{array}{c}\text { Suma de } \\
\text { cuadrados }\end{array}$ & Gl & $\begin{array}{c}\text { Media } \\
\text { cuadrática }\end{array}$ & F & Sig. \\
\hline \multirow{2}{*}{1} & Regresión & 786.086 & 17 & 46,240 & 6,889 &, $000^{\mathrm{b}}$ \\
& Residual & 322.171 & 48 & 6,712 & & \\
\cline { 2 - 7 } & Total & 1108.258 & 65 & & & \\
\hline
\end{tabular}

Fuente: Elaboración propia en base a los resultados obtenidos de GEM 
En la prueba F de Fisher se obtiene un valor de 6.889, este valor demuestra que las variables que han sido tomadas en cuenta para el modelo de Regresión, inciden en la generación de ecosistemas de innovación significativamente.

Concretando la investigación se observa que en el estudio de coeficientes (Tabla 5), se discriminan las variables del modelo de Regresión, para conocer los factores más significativos que hacen la diferencia en los distintos países de estudio, al momento de generar ecosistemas de innovación. A continuación, se muestra la fórmula aplicada para conocer los coeficientes.

$$
\text { Coeficiente }=\beta_{1}+\beta_{2}+\beta_{3}+\cdots+\beta_{p}
$$

Tabla 5 Coeficientes ${ }^{\mathrm{a}}$

\begin{tabular}{|c|c|c|c|c|c|}
\hline \multirow[t]{2}{*}{ Modelo } & \multicolumn{2}{|c|}{ Coeficientes no estandarizados } & \multirow{2}{*}{$\begin{array}{l}\begin{array}{l}\text { Coeficientes } \\
\text { tipificados }\end{array} \\
\text { Beta } \\
\end{array}$} & \multirow[t]{2}{*}{$\mathrm{T}$} & \multirow[t]{2}{*}{ Sig. } \\
\hline & $\mathrm{B}$ & Error típ. & & & \\
\hline Políticas gubernamentales & 2,848 & 2,145 & 261 & 1,328 & ,191 \\
\hline Apoyo del gobierno nacional & 1,595 & 1,395 & ,185 & 1,143 & 259 \\
\hline Apoyo del gobierno local & 2,115 & 1,796 & ,216 & 1,177 & ,245 \\
\hline Parques científicos e incubadoras & 6,876 & 1,493 &, 781 & 4,604 &, 000 \\
\hline Programas gubernamentales & $-3,430$ & 1,535 &,- 500 & $-2,234$ & 030 \\
\hline Educación primaria y secundaria &,- 527 & 2,701 &,- 038 &,- 195 & ,846 \\
\hline Educación universitaria & 2,005 & 1,951 & ,169 & 1,028 & ,309 \\
\hline Transferir conocimiento y tecnología & $-3,009$ & 2,185 &,- 305 & $-1,377$ & ,175 \\
\hline Acceso a nuevas tecnologías & 3,903 & 1,945 &, 354 & 2,007 &, 050 \\
\hline Pago de última tecnología & $-6,054$ & 1,426 &,- 718 & $-4,245$ &, 000 \\
\hline Subsidios tecnológicos & 214 & 2,070 & ,019 & ,103 & ,918 \\
\hline Financiamiento & $-2,826$ & 1,247 &,- 383 & $-2,266$ & 028 \\
\hline Experiencia empresarial & 1,479 & 1,625 & 188 & ,910 & ,367 \\
\hline Políticas (resumen) & $-5,565$ & 2,658 &,- 531 & $-2,094$ & 042 \\
\hline Programas gubernamentales (resumen) & 1), 076 & 048 & 180 & 1,582 & ,120 \\
\hline Transferencia de I+D (resumen) & $-1,023$ & 2,006 &,- 105 &,- 510 & ,612 \\
\hline Cultura (resumen) & 1,071 & 1,346 &, 163 & ,796 &, 430 \\
\hline
\end{tabular}

a. Variable dependiente: AÑO

Fuente: Elaboración propia en base a los resultados de la encuesta realizada por el GEM

Acorde con lo expuesto anteriormente, se han considerado los paradigmas de significancia y se aplica el estadístico de Durbin Watson para definir los factores relevantes del ecosistema de innovación de los 8 países de estudio. En primera instancia se selecciona las variables cuya significancia sea $>1$ para posteriormente descartar los factores con coeficientes negativos (coeficiente no estandarizado B), esto debido a que la mencionada prueba estadística indica que sí el coeficiente es negativo y la significancia mayor al error, entonces no se considera como parte del modelo de estudio. Obteniendo como resultado únicamente las variables con coeficientes $>1$ 
y con significancia $>0,05$. Finalmente, se determina que las variables favorables para un ecosistema son: subsidios tecnológicos, cultura empresarial, experiencia empresarial de los individuos, educación universitaria enfocada al emprendimiento, el apoyo al emprendimiento por parte del gobierno nacional y local; políticas y programas gubernamentales.

\section{Conclusiones}

El presente trabajo ha estudiado los factores relevantes de un ecosistema de innovación en ocho países tanto desarrollados como en vías de desarrollo, durante el periodo 2000-2013, analizando el papel desempeñado por diversos factores como son: educación, parques científicos, políticas, programas gubernamentales y tecnología.

Desde la perspectiva de los ecosistemas de innovación los factores analizados en este trabajo mantienen una estrecha relación con el desarrollo de actividades económicas. Luego de realizar un proceso de discriminación a través del estudio de coeficientes se determina que los factores más relevantes en los países de estudio son: subsidios tecnológicos, cultura empresarial, experiencia empresarial, educación universitaria, apoyo del gobierno nacional, apoyo de gobierno local, políticas y programas gubernamentales.

De entre los citados factores, los tres más relevantes para los países de Europa, EEUU y América latina se encuentran: tecnología, cultura y experiencia empresarial. Resulta de interés para estos países, la tecnología ya que tiene implicaciones relacionadas con la innovación disruptiva y esta a su vez con la generación de una ventaja competitiva, especialmente en los países desarrollados.

Adicionalmente dentro de los ecosistemas de innovación la cultura y experiencia empresarial son determinantes para quienes son parte de un contexto emprendedor, puesto que los individuos se motivan constantemente a través de los medios de interrelación y modelos empresariales a seguir.

Mientras en los países desarrollados el enfoque para iniciar una actividad empresarial no tiene implicaciones con formación y experiencia previa debido a que su contexto los ha dotado del conocimiento necesario, en América latina sucede lo contrario, el individuo aprende simultáneamente en el proceso emprendedor, circunstancia que impulsa a sus gobiernos a fortalecer los ecosistemas de innovación. 
Para los países analizados, la educación universitaria influye en el afianzamiento de las competencias de los potenciales emprendedores. También se destaca el impulso por parte del gobierno a través de políticas y programas que junto a las universidades promueven apoyo a las empresas de nueva creación, así como a la consolidación de las existentes mediante ecosistemas que a más de fortalecer su sustentabilidad, las prepara para la innovación (Al-Mubaraki \& Busler, 2017).

Este estudio no está libre de limitaciones, es el caso de los datos utilizados, las restricciones están relacionadas con la participación discontinua de los países que constan en la base de datos del GEM, periodo 2000-2013, lo que llevó a excluir a varios países de interés inicial.

\section{Referencias Bibliográficas}

Al-Mubaraki, H. M., \& Busler, M. (2017). Challenges and opportunities of innovation and incubators as a tool for knowledge-based economy. Journal of Innovation and Entrepreneurship, 6(1), 15. https://doi.org/10.1186/s13731-017-0075-y

Apa, R., Grandinetti, R., \& Sedita, S. R. (2017). The social and business dimensions of a networked business incubator: the case of H-Farm. Journal of Small Business and Enterprise Development, 24(2), 198-221. https://doi.org/10.1108/JSBED-07-2016-0103.

Arenal, A., Armuña, C., Villaverde, S. R., \& González, C. F. (2018). Ecosistemas emprendedores y startups, el nuevo protagonismo de las pequeñas organizaciones. Economía industrial, (407), 85-94.

Berlin Partner for Business and Technology. (2018). Capital de riesgo e incubadoras. Recuperado 10 de enero de 2019, de https:/www.businesslocationcenter.de/en/businesslocation/business-location/start-up-capital/venture-capital-incubators

Block, J. H., Fisch, C. O., \& Praag, M. van. (2017). The Schumpeterian entrepreneur: a review of the empirical evidence on the antecedents, behaviour and consequences of innovative entrepreneurship. Industry and Innovation, 24(1), 61-95. https://doi.org/10.1080/13662716.2016.1216397 
Buesa, M. (2006). El Sistema Nacional de Innovación en España. Recuperado de http://www.madrimasd.org/revista/revistaespeciall/articulos/buesa.asp

Camino, G. R. C., \& Aguilar, V. A. E. (2017). Emprendimiento e innovación en Ecuador, análisis de ecosistemas empresariales para la consolidación de pequeñas y medianas empresas. INNOVA Research Journal, 2(9), 73-87.

Carrillo, J., \& Contreras, O. (2015). Los enfoques analíticos y las políticas de innovación en el Norte de México. En Experiencias estatales y transfronterizas de innovación en México.

Casado, F. L., Siluk, J. C. M., \& Zampieri, N. L. V. (2012). Universidade empreendedora e desenvolvimento regional sustentável: proposta de um modelo. Revista de Administração da UFSM, 5(edição especial), 633-650.

Crevoisier, O. (2004). The Innovative Milieus Approach: Toward a Territorialized Understanding of the Economy? Economic Geography, 80(4), 367-379. https://doi.org/10.1111/j.19448287.2004.tb00243.x

Cruz, M., \& Arrieta, A. (2017). Ecosistemas de innovación en Latinoamérica. Recuperado 1 de enero de 2019, de https:/www.fomin.org/eses/PORTADAOld2015/Noticias/Comunicadosdeprensa/ArtMID/3819/ArticleID/12870/ Ecosistemas-de-innovaci\%C3\%B3n-en-Latinoam\%C3\%A9rica-.aspx

De los Ríos, S. S., Rodríguez, G. I., \& Sáenz-Díez, R. R. (2015). Emprendedores y capital riesgo en España: el caso de Fond-ICO Global. Revista Icade. Revista de Las Facultades de Derecho y Ciencias Económicas y Empresariales, 0(94), 31-65. https://doi.org/10.14422/icade.i94.y2015.002

Edquist, C., \& Lundvall, B.-Á. (1993). Comparing the Danish and Swedish Systems of Innovation. National Systems of Innovation. A Comparative Study, 265-291.

Fernández-Esquinas, M., Oostrom, M. van, \& Pinto, H. (2017). Key issues on innovation, culture and institutions: implications for SMEs and micro firms. European Planning Studies, 25(11), 1897-1907. https://doi.org/10.1080/09654313.2017.1364770 
Flores, L. P. (2017). Factores críticos que integran un modelo de gestión de un Parque Tecnológico para promover la competitividad regional en México. Recuperado de http://tesis.ipn.mx:8080/xmlui/handle/123456789/22563

Freeman, C. (1974). La teoría económica de la innovación industrial. España: Alianza. Recuperado de https://www.iberlibro.com/servlet/BookDetailsPL?bi=30044119379\&searchurl=tn\%3Dl a\%2Bteoria\%2Beconomica\%2Bde\%2Bla\%2Binnovacion\%2Bindustrial\%26sortby\%3D 20\%26an\%3Dfreeman\%2Bchristopher\&cm_sp=snippet-_-srp1-_-title4

Garzón, C. M. A., Ortiz, P. E., Acosta, P. J. C., Zárate, T. R. A., Pérez, R. I., Ramírez, G. M. T., ... Saiz, Á. J. M. (2015). Gestión de la sostenibilidad en el marco de las organizaciones. Universidad EAN. https://doi.org/10.21158/9789587563481

Gauipatín, C., \& Schwartz, L. (2014). Ecuador: Análisis del Sistema Nacional de Innovación: Hacia la consolidación de una cultura innovadora (Monografía del BID; 223) (p. 190). Banco Interamericano de Desarrollo. Recuperado de https://publications.iadb.org/es/publicacion/13821/ecuador-analisis-del-sistemanacional-de-innovacion-hacia-la-consolidacion-de-una

GEM Coordination Team. (2002, enero 17). Data Sharing and Public Release Policy. Recuperado de https://www.gemconsortium.org

Graham, C. R. (2006). Tecnología al servicio de la empresa y de la sociedad. Interfases, (1), 7-17.

ICEX. (2017). El ecosistema de las startups en Alemania y Reino Unido. Recuperado 10 de enero de 2019, de https://www.aulavirtualicex.es/seminario/el-ecosistema-de-las-startups-enalemania-y-reino-unido

Kantis, H. (Ed.). (2004). Desarrollo emprendedor: América Latina y la experiencia internacional. Washington DC.

Kumar, A., Kumar, R., Haque, M. R., Chowdhury, S. P., \& Islam, S. (2017). Entrepreneurial Networks and Knowledge Transfer: The Moderating Role of Incubator/Accelerator 
Affiliation. Asian Economic and Financial Review, 7(11), 1093-1107. https://doi.org/10.18488/journal.aefr.2017.711.1093.1107

Lederman, D., Messina, J., Pienknagura, S., \& Rigolini, J. (2014). El emprendimiento en America Latina: muchas empresas y poca innovacion (No. 83837) (pp. 1-181). The World Bank. Recuperado de http://documentos.bancomundial.org/curated/es/154071468242382906/Elemprendimiento-en-America-Latina-muchas-empresas-y-poca-innovacion

Leydesdorff, L. (2006). The Knowlwdge-based economy: modeled, measured, simulated. Boca Raton, Florida: Universal Publishers.

Lundvall, B.-Á. (Ed.). (1992). National systems of innovation: Towards a Theory of innovation and interactive learning. London: Pinter Publishers.

Mahlmann, K. L., Rodrigues, E., Ferrari, A. G., \& Bueno, M. B. (2014). Universities and Incubators: Key Entrepreneurship and Socioeconomic Development Driving Factors. Independent Journal of Management \& Production, 5(4), 947-965.

Malecki, E. J., \& Spigel, B. (2017). Innovation and entrepreneurship. Edward Elgar Publishing. Recuperado de https://www.elgaronline.com/view/9781782548515.00050.xml

Martínez, D. A., González, A. N., \& Nieto, M. (2015). LA INNOVACIÓN SOCIAL COMO MOTOR DE LA CREACIÓN DE EMPRESAS. Universia Business Review, 0(47). Recuperado de https://ubr.universia.net/article/view/1505/innovacion-social-motor-lacreacion-empresas

Metcalfe, S. (1995). Technology Systems and Technology Policy in an Evolutionary Framework (Vol. 19). Oxford University Press. https://doi.org/10.1093/oxfordjournals.cje.a035307

Moore, J. F. (1993). Predators and Prey: A New Ecology of Competition. Harvard Bussiness Review, 3, 75-86.

Moore, J. F. (1999). The Death of Competition - Leadership \& Strategy in the Age of Business Ecosystems. Wiley. 
Nelson, R. R. (1993). National Innovation Systems: A Comparative Analysis. New York: Oxford University Press.

Nicolopoulou, K., Karatas- Ozkan, M., Nummann, M., \& Vas, C. (2015). An incubation perspective on social innovation: the London Hub as a social. R\&D Management. Recuperado de https://strathprints.strath.ac.uk/54506/

Ochoa, Á. M. B., Quevedo, A. Y., \& Valdés, S. M. (2007). Innovación, tecnología y gestión tecnológica. Revista Cubana de Información en Ciencias de la Salud, 16(4), 8.

Ochoa, H. V., Hinojosa, D. R. D., \& Hinojosa, D. S. M. (2018). Impacto de la creación de Ecosistemas de Innovación Social en las Instituciones de Educación Superior en el Ecuador. Yachana Revista Científica, $0(0)$. Recuperado de http://revistas.ulvr.edu.ec/index.php/yachana/article/view/441

OECD. (2016). Startup América Latina 2016. Recuperado de https:/www.oecdilibrary.org/content/publication/9789264265141-es

Patel, P., \& Pavitt, K. (1994). National Innovation Systems: Why They Are Important, And How They Might Be Measured And Compared (Vol. 3). https://doi.org/10.1080/10438599400000004

Peña, I., Guerrero, M., González, P. J. L., \& Montero, J. (2017). Global Entrepreneurship Monitor: informe GEM España 2017-2018 (anual). Madrid. Recuperado de https://www.gemconsortium.org

Pocorey, C. L. F., \& Ayabe, M. (2016). ANÁLISIS COMPARATIVO DEL ENTORNO DE INVESTIGACIÓN EN LAS UNIVERSIDADES DE ALEMANIA, FINLANDIA, JAPÓN Y BOLIVIA. Revista Tecnológica, 15.

ProChile, O. C. de C. en G. (2014). Ecosistemas de Emprendimiento e Innovación en México. Recuperado de https://www.prochile.gob.cl/documento-biblioteca/ecosistemas-deemprendimiento-e-innovacion-en-mexico/ 
ProChile, O. C. de C. en L. Á. (2014). Ecosistemas de Emprendimiento e Innovación en Los Ángeles, Estados Unidos. Recuperado 10 de enero de 2019, de https://www.prochile.gob.cl/documento-biblioteca/ecosistemas-de-emprendimiento-einnovacion-en-los-angeles-estados-unidos/

Ramírez, S. M. del P., \& García, V. M. (2010). The University-Company- State Alliance: a strategy to promote innovation. Revista EAN, (68), 112-133.

Ribeiro, P. W., Vieira, B. G., Bent, H. P., Lima, R. R., \& Meister, M. H. (2015). The relationship between the competencies of a technology park and the competitiveness of its resident IT enterprises: a case analysis at TECNOPUC. Revista de Administração Da Universidade Federal de Santa Maria, 8(4), 540-560.

Rincón, C. E. L. (2004). El sistema nacional de innovación: Un análisis teórico-conceptual. Opción, 20(45), 94-117.

Ríos, M. Á. R., \& Hernández, R. C. (2003). Los Sistemas De Innovación Nacionales Y La Teoría Del Desarrollo. Problemas del Desarrollo. Revista Latinoamericana de Economía, 34(134), 9-31.

Rossi, F. (2010). The governance of university-industry knowledge transfer (Vol. 13). https://doi.org/10.1108/14601061011040230

Salvador, E. (2008). Políticas rexionais de innovación no Reino Unido: a nova relación entre a industria e as institucións de educación superior (HEI) e o papel dos parques científicos. Revista galega de economía: Publicación Interdisciplinar da Facultade de Ciencias Económicas e Empresariais, 17(1), 131-156.

Smith, H. L. (2018). Entrepreneurship Policies and the Development of Regional Innovation Systems: Theory, Policy and Practice. En A. Isaksen, R. Martin, \& M. Trippl (Eds.), New Avenues for Regional Innovation Systems - Theoretical Advances, Empirical Cases and Policy Lessons (pp. 239-256). Cham: Springer International Publishing. https://doi.org/10.1007/978-3-319-71661-9_12 
Sternberg, R., Wallsich, M., Gorynia-Pfeffer, N., Von Bloh, J., \& Baharian, A. (2017). Global Entrepreneurship Monitor GEM: Country Report Germany 2017-2018. Recuperado de https://www.gemconsortium.org

Vincens, L., \& Grullon, S. (2011). Innovación y emprendimiento: Un modelo basado en el desarrollo del emprendedor. Banco Interamericano de Desarrollo. Recuperado de https://publications.iadb.org/es/publicacion/15039/innovacion-y-emprendimiento-unmodelo-basado-en-el-desarrollo-del-emprendedor

Wang, J. (2018). Innovation and government intervention: A comparison of Singapore and Hong Kong. Research Policy, 47(2), 399-412. https://doi.org/10.1016/j.respol.2017.12.008

Witte, P., Slack, B., Keesman, M., Jugie, J.-H., \& Wiegmans, B. (2017). Facilitating start-ups in port-city innovation ecosystems: A case study of Montreal and Rotterdam. Journal of Transport Geography. https://doi.org/10.1016/j.jtrangeo.2017.03.006

Wood, D., Wilson, C., \& García, A. (2014). Fomentando la innovación en México: Ideas del Foro de Innovación de Alto Nivel para Creadores de Política. Washington. Recuperado de www.wilsoncenter.org 